\title{
$\angle S$ Research Square \\ Incidence and Risk Factors of Infusion Reactions in Breast Cancer Patients Administered Pertuzumab Combination Chemotherapy
}

\section{Yusuke Tabuchi}

Kyoto Pharmaceutical University: Kyoto Yakka Daigaku https://orcid.org/0000-0002-4283-8835

\section{Masayuki Tsujimoto}

Kyoto Pharmaceutical University: Kyoto Yakka Daigaku

\section{Kosuke Yamamoto}

Kyoto Prefectural University of Medicine: Kyoto Furitsu Ika Daigaku

\section{Tadashi Kosaka}

Kyoto Prefectural University of Medicine: Kyoto Furitsu Ika Daigaku

\section{Koichi Sakaguchi}

Kyoto Prefectural University of Medicine: Kyoto Furitsu Ika Daigaku

\section{Naoya Dobuchi}

Kyoto Prefectural University of Medicine: Kyoto Furitsu Ika Daigaku

\section{Kohshi Nishiguchi}

Kyoto Pharmaceutical University: Kyoto Yakka Daigaku

Keisuke Shikata ( $\square$ kshikata@koto.kpu-m.ac.jp )

Kyoto Prefectural University of Medicine: Kyoto Furitsu lka Daigaku https://orcid.org/0000-0002-76012185

\section{Research Article}

Keywords: infusion reaction, pertuzumab, risk factor, breast cancer

Posted Date: March 1st, 2022

DOI: https://doi.org/10.21203/rs.3.rs-1294435/v1

License: (c) (i) This work is licensed under a Creative Commons Attribution 4.0 International License.

Read Full License 


\section{Abstract}

Purpose: Pertuzumab (Per) is a humanized monoclonal antibody used in combination with trastuzumab (Tra) in the treatment of human epidermal growth factor receptor-2 (HER2)-positive breast cancer. The administration of biologics, such as Tra and Per, frequently causes infusion reactions (IRs) with fever and chills. The purpose of this study was to clarify the characteristics of and risk factors for IRs in Tra+Per combination therapy.

Methods: Between March 2013 and December 2019, 65 breast cancer patients who started Tra+Per combination therapy and 106 breast cancer patients who started Tra therapy alone were included in the study. The severity of IRs was graded according to the Common Terminology Criteria for Adverse Events (CTCAE) version 5.0.

Results: The incidence of IRs in the Tra+Per group was $47.7 \%$ (31/65) and was significantly higher than that in the Tra alone group $(18.9 \%, 20 / 106)$, with a risk ratio of $2.53(p<0.001)$. The severity of IRs in the Tra+Per group (Grade 1, 9 patients; Grade 2, 22 patients) was significantly higher than that in the Tra alone group (grade 1, 16 patients; grade 2,4 patients) $(p<0.001)$. Lymphocyte counts were significantly different between the IR and non-IR groups in patients receiving Tra+Per combination therapy (univariate analysis, $p=0.005$; multivariate analysis, $p=0.029$ ).

Conclusion: Our study indicates that the concomitant use of Per increases the risk of IRs caused by Tra, and the risk of IRs is higher in patients with higher lymphocyte counts.

\section{Introduction}

Pertuzumab is a humanized monoclonal antibody indicated for the treatment of human epidermal growth factor receptor-2 (HER2)-positive breast cancer and is used in combination with trastuzumab. Triple combination therapy with trastuzumab, pertuzumab, and docetaxel (Tra+Per+DTX) has shown effectiveness in the treatment of HER2-positive metastatic recurrent breast cancer [1], and combination therapy with trastuzumab and pertuzumab (Tra+Per) has shown effectiveness in the treatment of HER2positive early-stage breast cancer [2].

The administration of biologics such as trastuzumab and pertuzumab frequently causes infusion reactions with fever and chills. Infusion reactions associated with the administration of biologics frequently occur at the time of initial administration, and are considered to be acute-phase reactions caused by the cytokines released from tumor cells and effector cells that accumulate around the tumor [3]. Some patients taking biologics have been reported to show severe infusion reactions [4]; therefore, medical workers should carefully monitor patients after administration. The occurrence of infusion reactions also complicates the management of chemotherapy because the additional treatments necessitated by these reactions require more staff time and entail higher human resource costs [5]. 
In cases involving trastuzumab monotherapy, infusion reactions occur in $16.2 \%$ of patients, and high body mass index (BMI), stage IV (clinical stage) disease, and non-premedication have been reported to be risk factors for trastuzumab-induced infusion reactions [6]. In addition, the APHINITY trial has reported that the incidence of infusion reactions is $20.9 \%$ in HER2-positive early-stage breast cancer patients treated with Tra+Per. A comparison of these findings suggests that the addition of pertuzumab may have little effect on the incidence of infusion reactions in trastuzumab therapy. However, only a few reports have described the incidence of infusion reactions with Tra+Per combination therapy, and it remains unclear whether the comparative result is applicable in clinical practice with diversity. Information on the incidence of infusion reactions and the related risk factors will be beneficial for patients undergoing Tra+Per combination therapy, since it will allow adaptation of treatment regimens according to the individual risk of infusion reactions in each patient.

The purpose of this study was to identify the risk factors for infusion reactions in Tra+Per combination therapy by comparing the incidence and characteristics of infusion reactions in HER2-positive breast cancer patients treated with Tra+Per combination therapy and trastuzumab monotherapy.

\section{Patients And Methods}

\subsection{Study period and participants}

Sixty-nine breast cancer patients who started chemotherapy with trastuzumab and pertuzumab at the Kyoto Prefectural University of Medicine Hospital and Kyoto Prefectural University of Medicine Northern Medical Center between October 2013 and December 2019 were included in this study. This study also included 111 breast cancer patients who started chemotherapy with trastuzumab without pertuzumab at the same hospitals during the same period. However, nine patients who started treatment at other hospitals were excluded from this study because infusion reactions with biologics are known to occur mainly at the time of first administration [7].

In patients receiving Per+Tra therapy, $840 \mathrm{mg} /$ body of pertuzumab was administered intravenously for 60 min, and after a 60-minute follow-up assessment for adverse events such as infusion reactions, $8 \mathrm{mg} / \mathrm{kg}$ of trastuzumab was administered intravenously over $90 \mathrm{~min}$ (initial dose). The second and subsequent doses were $420 \mathrm{mg} /$ body pertuzumab were administered intravenously over $30 \mathrm{~min}$, followed by a 30minute follow-up period, and $6 \mathrm{mg} / \mathrm{kg}$ trastuzumab was administered intravenously over $30 \mathrm{~min}$. Provided that more than six weeks had passed since the previous administration, the same dose as the initial dose was administered.

\subsection{Extraction of variables}

Sex, premedication (dexamethasone), age, height, weight, BMI, stage classification, receptor status, and drug administration history were investigated as patient background factors. Laboratory data (lactate dehydrogenase [LDH], alanine aminotransferase [ALT], aspartate aminotransferase [AST], total bilirubin [T-Bil], creatinine [CRE], hemoglobin [HGB], and albumin [ALB] levels, neutrophil count, and lymphocyte 
count) immediately before the first administration of pertuzumab and trastuzumab were collected. Infusion reactions were defined as adverse events of chills, fever, fatigue, erythema, hypertension, headache, nausea, palpitations, and dyspnea occurring within $24 \mathrm{~h}$ after administration of trastuzumab. The severity of infusion reactions was graded according to the Common Terminology Criteria for Adverse Events (CTCAE) version 5.0. The survey items were extracted from hospital electronic medical records.

\subsection{Statistical analysis}

The Mann-Whitney U test was used to compare physical information (age, height, body weight, BMI) in the Tra+Per and trastuzumab alone (Tra alone) groups and laboratory data in the IR and non-IR groups. Fisher's exact test was used to compare patient background in the Tra+Per and Tra alone groups and the association between infusion reaction occurrence and patient background factors. For the analysis of factors influencing the occurrence of infusion reactions, multivariate analysis (logistic regression analysis) was performed for BMI and stage IV [6], which have been reported to influence the occurrence of infusion reactions in trastuzumab monotherapy, as well as for factors that showed a $p$ value $<0.2$ in the univariate analysis in this study. All statistical analyses were performed using EZR [8], which is used for R. More precisely, it is a modified version of R commander designed to add statistical functions frequently used in biostatistics. The level of significance was set at less than 0.05 , in each case.

\section{Result}

\subsection{Participants}

Sixty-nine patients in the Tra+Per group (61 at Kyoto Prefectural University of Medicine Hospital and eight at Kyoto Prefectural University of Medicine Northern Medical Center) and 111 patients in the Tra alone group (98 at Kyoto Prefectural University of Medicine Hospital and 13 at Kyoto Prefectural University of Medicine Northern Medical Center) were included in this study. Four patients in the Tra+Per group and five patients in the Tra alone group were excluded from this study because they started receiving trastuzumab at other hospitals.

\subsection{Patient characteristics}

Table 1 presents the characteristics of the patients in this study. Premedication (dexamethasone) $(p<$ $0.001)$ and stage classification $(p<0.001)$ were significantly different between the Tra+Per and Tra alone groups. The two groups showed no differences in sex, age, height, body weight, and receptor status. Most patients in the Tra+Per group received docetaxel (78.5\%), and patients in the trastuzumab alone group mainly received docetaxel (56.6\%) and cyclophosphamide (54.7\%). 
Table 1

Patient characteristics in this study

\begin{tabular}{|c|c|c|c|}
\hline & $\begin{array}{l}\text { Tra+Per } \\
(n=65)\end{array}$ & $\begin{array}{l}\text { Tra alone } \\
(n=106)\end{array}$ & $P$ value \\
\hline Sex (Male / Female) & $0 / 65$ & $2 / 104$ & $0.526^{\text {a) }}$ \\
\hline Premedications (Yes / No) & $1 / 64$ & $78 / 28$ & $<0.001^{a), *}$ \\
\hline Age (y) & $61(35-80)$ & $57(33-83)$ & $0.115^{b)}$ \\
\hline Height (cm) & $157.0(135.5-170.0)$ & $156.0(139.9-171.4)$ & $0.438^{b)}$ \\
\hline Body weight (kg) & $52.0(38.5-81.7)$ & $55.0(37.3-104)$ & $0.247^{b)}$ \\
\hline BMI & $21.7(15.4-30.8)$ & $21.7(15.4-30.8)$ & $0.657^{b)}$ \\
\hline \multicolumn{4}{|l|}{ Stage } \\
\hline$\square$ & $14(21.5)$ & $49(46.2)$ & $<0.001^{a), *}$ \\
\hline$\square$ & $26(40.0)$ & $37(34.9)$ & \\
\hline$\square$ & $11(16.9)$ & $18(17.0)$ & \\
\hline$\square$ & $14(21.5)$ & $2(2.0)$ & \\
\hline \multicolumn{4}{|l|}{ Receptor status } \\
\hline ER positive & $34(52.3)$ & $70(66.0)$ & 0.079 a) \\
\hline PR positive & $30(46.2)$ & $42(40.0)$ & $0.428^{a)}$ \\
\hline HER positive & $65(100)$ & $106(100)$ & - \\
\hline \multicolumn{4}{|c|}{ Concurrent anticancer therapy } \\
\hline Docetaxel & $51(78.5)$ & $60(56.6)$ & \\
\hline Nab-paclitaxel & $1(1.5)$ & $17(16.0)$ & \\
\hline Eribrin & $1(1.5)$ & $3(2.8)$ & \\
\hline Cyclophosphamide & $0(0.0)$ & $58(54.7)$ & \\
\hline
\end{tabular}

All data were reported as median (range) or $\mathrm{n}(\%)$

Tra, trastuzumab; Per, pertuzumab; $B M I$, body mass index; $E R$, estrogen receptor; $P R$, progesterone receptor; $H E R$, human epidermal growth factor receptor; nab-PTX, nanoparticle albumin-bound paclitaxel

a) Fisher's exact test; ${ }^{b)}$ Mann-Whitney U test $\left({ }^{\star} P<0.05\right)$. 


\begin{tabular}{|c|c|c|c|}
\hline & $\begin{array}{l}\text { Tra+Per } \\
(n=65)\end{array}$ & $\begin{array}{l}\text { Tra alone } \\
(n=106)\end{array}$ & $P$ value \\
\hline Paclitaxel & $0(0.0)$ & $9(8.5)$ & \\
\hline None & $12(18.5)$ & $17(16.0)$ & \\
\hline Pretreatment with Tra & $27(41.5)$ & - & \\
\hline \multicolumn{4}{|c|}{ All data were reported as median (range) or $\mathrm{n}(\%)$} \\
\hline \multicolumn{4}{|c|}{$\begin{array}{l}\text { Tra, trastuzumab; Per, pertuzumab; } B M I \text {, body mass index; } E R \text {, estrogen receptor; } P R \text {, progesterone } \\
\text { receptor; } H E R \text {, human epidermal growth factor receptor; nab-PTX, nanoparticle albumin-bound } \\
\text { paclitaxel }\end{array}$} \\
\hline
\end{tabular}

\subsection{Incidence and status of infusion reactions}

The incidence of infusion reactions in the Tra+Per group was $47.7 \%$ (31/65), which was significantly higher than that in the Tra alone group $(18.9 \%, 20 / 106)$ (Fig. 1, p < 0.001). The risk ratio of infusion reactions in the Tra+Per group was 2.53 (Fig. 1). Table 2 shows the characteristics of infusion reactions in the patients. The incidence of infusion reactions was high at the first dose in both the Tra+Per group (31/31) and the Tra alone group (19/20). The severity of infusion reactions in the Tra+Per group (Grade 1, 9 patients; Grade 2, 22 patients) was significantly higher than that in the Tra alone group (grade 1, 16 patients; grade 2, 4 patients; $p<0.001$ ). The symptoms of infusion reactions varied, including chills, fever, headache, nausea, and palpitations. All infusion reactions did not occur during pertuzumab administration but during or after trastuzumab administration. 
Table 2

Severity, symptoms, and onset time in patients with infusion reactions

\begin{tabular}{|c|c|c|c|}
\hline & $\begin{array}{l}\text { Tra+Per } \\
(n=31)\end{array}$ & $\begin{array}{l}\text { Tra alone } \\
(n=20)\end{array}$ & $P$ value \\
\hline \multicolumn{4}{|l|}{$\mathrm{n}(\%)$} \\
\hline First dose & $31(100.0)$ & $19(95.0)$ & 0.392 \\
\hline Subsequent doses & $5(16.1)$ & $1(5.0)$ & 0.384 \\
\hline \multicolumn{4}{|l|}{ Severity } \\
\hline Grade 1 & $9(29.0)$ & $16(80.0)$ & \multirow[t]{3}{*}{$<0.001^{*}$} \\
\hline Grade 2 & $22(71.0)$ & $4(20.0)$ & \\
\hline$\geqq$ Grade 3 & $0(0.0)$ & $0(0.0)$ & \\
\hline \multicolumn{4}{|l|}{ Symptoms } \\
\hline Chills & $22(71.0)$ & $8(40.0)$ & - \\
\hline Fever & $19(61.3)$ & $6(30.0)$ & \\
\hline Headache & $7(22.6)$ & $3(15.0)$ & \\
\hline Nausea & $3(9.7)$ & $4(20.0)$ & \\
\hline Palpitation & $0(0.0)$ & $3(15.0)$ & \\
\hline \multicolumn{4}{|l|}{ Onset time } \\
\hline During Pertuzumab & $0(0.0)$ & - & 0.345 \\
\hline During Trastuzumab & $23(74.2)$ & $12(60.0)$ & \\
\hline After Trastuzumab & $7(22.6)$ & $8(40.0)$ & \\
\hline Unknown & $1(3.2)$ & $0(0.0)$ & \\
\hline \multicolumn{4}{|c|}{ Tra, trastuzumab; Per, pertuzumab } \\
\hline Fisher's exact test $\left({ }^{\star} P\right.$ & $0.05)$ & & \\
\hline
\end{tabular}

\subsection{Management and subsequent therapy in patients with infusion reactions}

The management of therapy after the infusion reactions is shown in Table 3. The symptoms of all patients improved within the same day in both groups. Some patients took acetaminophen, loxoprofen, 
and corticosteroids for infusion reactions. Most patients in both groups (83.9\% in the Tra+Per group and $90 \%$ in the Tra alone group) were rechallenged and completed the therapy within the same day. Only one patient could not resume therapy in the Tra+Per group.

Table 3

Management and subsequent therapy in patients with infusion reactions

\begin{tabular}{|lll|}
\hline & $\begin{array}{l}\text { Tra+Per } \\
(\mathbf{n}=\mathbf{3 1})\end{array}$ & $\begin{array}{l}\text { Tra alone } \\
(\mathbf{n}=\mathbf{2 0})\end{array}$ \\
\hline $\mathrm{n}(\%)$ & & \\
\hline Infusion stopped & $26(83.9)$ & $8(40.0)$ \\
\hline Symptom resolution & $31(100.0)$ & $20(100.0)$ \\
\hline Medication given for reation & $19(61.3)$ & $4(20.0)$ \\
\hline Acetaminophen & $14(45.2)$ & $3(15.0)$ \\
\hline Loxoprofen & $5(16.1)$ & $0(0.0)$ \\
\hline Antihistamine & $3(9.7)$ & $0(0.0)$ \\
\hline Adrenal corticosteroid & $3(9.7)$ & $1(5.0)$ \\
\hline Rechallenge same day & $26(83.9)$ & $18(90.0)$ \\
\hline Received subsequent therapy & $30(96.8)$ & $20(100.0)$ \\
\hline
\end{tabular}

\subsection{Risk factors of infusion reactions in patients taking with Tra+Per therapy}

Comparison of physical information and laboratory data between the patients that showed infusion reaction and those that did not show infusion reactions after receiving Tra+Per combination therapy is presented in Table 4. Only lymphocyte counts were significantly different between the two groups $(p=$ 0.005). The results of the multivariate analysis are presented in Table 5. Multivariate analysis indicated that high lymphocyte counts just before therapy were a risk factor for infusion reactions in Tra+Per combination therapy (odds ratio, 3.08; $95 \%$ confidence interval, 1.13-8.44; $p=0.029$ ). 
Table 4

Risk factors for the onset of infusion reactions after pertuzumab administration

\begin{tabular}{|c|c|c|c|}
\hline & $\begin{array}{l}\text { IR } \\
(n=31)\end{array}$ & $\begin{array}{l}\text { Non-IR } \\
(n=34)\end{array}$ & $P$ value \\
\hline Age (y) & $63(35-80)$ & $61(41-76)$ & $0.708^{b)}$ \\
\hline Height (cm) & $157(143.4-163)$ & $157.7(135.5-170)$ & $0.338^{b)}$ \\
\hline Body weight (kg) & $53(42.7-81.7)$ & $52.0(38.5-72.4)$ & $0.327^{b)}$ \\
\hline BMI & $21.0(17.7-30.8)$ & $21.0(15.4-28.7)$ & $0.110^{b)}$ \\
\hline Pretreatment with Tra & $10(32.3)$ & $17(50.0)$ & $0.208^{b)}$ \\
\hline \multicolumn{4}{|l|}{ Stage } \\
\hline 口 & $6(19.4)$ & $8(23.5)$ & $0.815^{\text {a) }}$ \\
\hline$\square$ & $14(45.2)$ & $12(35.3)$ & \\
\hline Q & $4(12.9)$ & $7(20.6)$ & \\
\hline Q & $7(22.6)$ & $7(20.6)$ & \\
\hline $\mathrm{LDH}(\mathrm{U} / \mathrm{L})$ & $193(144-403)$ & $192(120-315)$ & $0.818^{b)}$ \\
\hline $\operatorname{ALT}(\mathrm{U} / \mathrm{L})$ & $16(6-45)$ & $17.5(4-44)$ & $0.798^{b)}$ \\
\hline AST (U/L) & $21(13-46)$ & $22(13-58)$ & $0.968^{b)}$ \\
\hline T-Bil (mg/dL) & $0.57(0.37-1.11)$ & $0.60(0.20-1.73)$ & $0.722^{b)}$ \\
\hline CRE (mg/dL) & $0.63(0.40-1.15)$ & $0.60(0.3-1.03)$ & $0.163^{b)}$ \\
\hline $\mathrm{CCr}(\mathrm{ml} / \mathrm{min})$ & $80.6(36.5-212.2)$ & $81.4(42.4$ - 267.6) & $0.597^{b)}$ \\
\hline eGFR (mL/min/1.73m2) & $72.3(35.9-125.7)$ & $77.5(41.7$ - 184.3) & $0.117^{b)}$ \\
\hline $\mathrm{HGB}(\mathrm{g} / \mathrm{dL})$ & $12.3(8.5-15.8)$ & $12.7(8.5-15)$ & $0.921^{b)}$ \\
\hline ALB $(g / d L)$ & $4.1(3.6-4.8)$ & $4.3(3.1-4.8)$ & $0.091^{b)}$ \\
\hline
\end{tabular}

All data are reported as median (range) or $\mathrm{n}(\%)$

$B M I$, body mass index; Tra, trastuzumab; $L D H$, lactic acid dehydrogenase; $A L T$, alanine aminotransferase; $A S T$, aspartate aminotransferase; $T$-Bil, total bilirubin; $C R E$, creatinine; $C C r$, creatinine clearance; $e G F R$, estimated glomerular filtration rate; $H G B$, hemoglobin; $A L B$, albumin

a) Fisher's exact test; b) Mann-Whitney U test $\left({ }^{\star} P<0.05\right)$. 


\begin{tabular}{|c|c|c|c|}
\hline & $\begin{array}{l}\text { IR } \\
(n=31)\end{array}$ & $\begin{array}{l}\text { Non-IR } \\
(\mathrm{n}=34)\end{array}$ & $P$ value \\
\hline Neutrophil $\left(10^{3} / \mu \mathrm{L}\right)$ & $2.97(1.03-6.73)$ & $2.67(1.22-6.45)$ & $0.419^{b)}$ \\
\hline Lymphocyte $\left(10^{3} / \mu \mathrm{L}\right)$ & $1.68(0.5-3.58)$ & $1.20(0.41-2.97)$ & $0.005^{b), *}$ \\
\hline \multicolumn{4}{|c|}{ All data are reported as median (range) or $\mathrm{n}(\%)$} \\
\hline \multicolumn{4}{|c|}{$\begin{array}{l}B M I \text {, body mass index; Tra, trastuzumab; } L D H \text {, lactic acid dehydrogenase; } A L T \text {, alanine } \\
\text { aminotransferase; } A S T \text {, aspartate aminotransferase; } T \text {-Bil, total bilirubin; } C R E \text {, creatinine; } C C r \text {, } \\
\text { creatinine clearance; } G G R \text {, estimated glomerular filtration rate; } H G B \text {, hemoglobin; } A L B \text {, albumin }\end{array}$} \\
\hline \multicolumn{4}{|c|}{ a) Fisher's exact test; b) Mann-Whitney U test $\left({ }^{\star} P<0.05\right)$. } \\
\hline
\end{tabular}

Table 5

Multivariate analysis of risk factors and their relationships with infusion reactions

\begin{tabular}{|lllll|}
\hline & Contrast & Odds ratio & $95 \%$ Cl & P value \\
\hline eGFR & 1-unit increase & 0.984 & $0.958-101$ & 0.216 \\
\hline BMI & 1-unit increase & 1.060 & $0.902-1.25$ & 0.462 \\
\hline Stage & IV vs. others & 0.713 & $0.171-2.97$ & 0.640 \\
\hline ALB & 1-unit increase & 0.270 & $0.0433-1.69$ & 0.162 \\
\hline Lymphocyte & 1-unit increase & 3.080 & $1.13-8.44$ & $0.029^{*}$ \\
\hline logistic regression analysis ( $P<0.05)$ & & & \\
\hline $\begin{array}{l}\text { Cl, confidence interval; } \text { eGFR, estimated glomerular filtration rate; } B M I, \text { body mass index; } \\
\text { albumin. }\end{array}$ & & & \\
\hline
\end{tabular}

\section{Discussion}

This study indicated that co-administration of pertuzumab increased the incidence and severity of infusion reactions caused by the administration of trastuzumab. In addition, this study showed that high lymphocyte counts just before therapy were a risk factor for infusion reactions in patients receiving Tra+Per combination therapy. These findings provide valuable information for the prevention of infusion reactions during Tra+Per combination therapy.

The incidence of infusion reactions in patients receiving Tra+Per was $47.7 \%$, which was significantly higher than that in patients receiving Tra alone (18.9\%), with a risk ratio of 2.53 (Fig. 1). The incidence of infusion reactions for trastuzumab monotherapy (18.9\%) was similar to the results in a previous report (16.2\%) [6], indicating that the incidence of infusion reactions during trastuzumab monotherapy was 
reasonable in this study. In patients receiving Tra+Per, infusion reactions were not observed during pertuzumab treatment, but they appeared during or after trastuzumab administration (Table 2). The incidence $(13.0 \%)$ of infusion reactions for Tra+Per combination therapy in the CLEOPATRA trial for metastatic recurrent breast cancer was lower than that in the neoadjuvant and adjuvant trials (18.6\%-25.0\%) for early-stage breast cancer [9] and in our study (47.7\%). One reason for the low incidence in the CLEOPATRA trial is the long interval between pertuzumab (day 1 ) and Tra+DTX (day 2). Thus, pertuzumab did not increase the risk of infusion reactions associated with trastuzumab in the CLEOPATRA trial, since it was not administered on the same day. Therefore, our results suggest that coadministration of pertuzumab increases the incidence and severity of infusion reactions caused by trastuzumab.

Our results indicate that the pretreatment lymphocyte counts in patients who showed infusion reactions during the Tra+Per combination therapy were significantly higher than those in patients who did not show infusion reactions (Table 4). Multivariate analysis identified lymphocyte counts just before treatment as a risk factor for the development of infusion reactions in Tra+Per combination therapy (Table 5). Patients taking rituximab, another biologic drug used for chronic lymphocytic leukemia, and showing lymphocyte counts greater than $5.0 \times 10^{4} / \mu \mathrm{L}$ have been reported to show a significantly higher rate of severe symptoms than patients taking rituximab with low lymphocyte counts [10]. Thus, the infusion reactions caused by rituximab are more likely to become severe in patients with high lymphocyte counts. This is thought to be because the number of lymphocytes increases as the disease progresses, and the incidence and severity of infusion reactions increase accordingly because CD20-positive B cells can release cytokines during death. In contrast, pertuzumab and trastuzumab activate antibody-dependent cytotoxicity (ADCC) through effector cells such as macrophages and natural killer cells (NK cells) [11]. Activated NK cells, one of the lymphocytes, can produce cytokines such as tumor necrosis factor alpha, so higher numbers of NK cells release more cytokines, which may increase the incidence and severity of infusion reactions. Thus, a novel finding of the present study is that the number of circulating lymphocytes is important for the incidence of infusion reactions not only in hematopoietic tumors but also in solid tumors.

All patients in the Tra+Per combination therapy group who showed infusion reactions during the second and subsequent doses also showed infusion reactions after the first dose. In the Tra alone group, 93.8\% of patients showing infusion reactions have been reported to develop infusion reactions at the first dose [6]. In addition, high-dose patients (maintenance therapy at $4 \mathrm{mg} / \mathrm{kg}$ after the initial dose of $8 \mathrm{mg} / \mathrm{kg}$ ) had higher rates of fever ( $45 \%$ vs. $36 \%$ ) and chills ( $40 \%$ vs. $22 \%$ ) than low-dose patients (maintenance therapy at $2 \mathrm{mg} / \mathrm{kg}$ after an initial dose of $4 \mathrm{mg} / \mathrm{kg}$ ) [12]. On the basis of these results, we speculate that the incidence of infusion reactions is higher after the first dose because of the use of loading doses of both trastuzumab and pertuzumab. Therefore, special attention should be paid to infusion reactions at the first dose in the Tra+Per combination therapy.

Our study did not extract BMI as a risk factor of infusion reactions (Table 4), but high BMI has been reported to increase the risk of infusion reactions with trastuzumab monotherapy [6]. Only $23.1 \%$ of the 
patients in our study had a high BMI ( 25 or more), which was markedly less than the proportion in a previous study (69.5\%), and one reason why the influence of BMI was not observed may be the difference in patient background. In addition, this study did not extract stage classification as a risk factor for infusion reaction (Table 4), unlike previous reports on trastuzumab monotherapy [6]. The incidence of infusion reactions in patients with stage IV disease was $50 \%(7 / 14)$. The incidence of infusion reactions in stage IV patients pretreated with trastuzumab $(1 / 6,16.8 \%)$ tended to be lower than that in patients not pretreated with trastuzumab $(6 / 8,75 \% ; p=0.103)$ in this study. Therefore, one reason why the influence of stage classification is different could be pretreatment with trastuzumab.

Prediction of infusion reactions on the basis of the lymphocyte count, which was identified as a risk factor for infusion reactions in this study, will enable consideration of prophylactic antipyretic analgesic medication and enhanced care tailored to individual patient risk. In the future, we hope to conduct a study with a larger patient population and investigate the benefit of premedication in Tra+Per combination therapy and the relationship between infusion reaction occurrence and therapeutic effect so that chemotherapy for breast cancer patients can be provided more safely and effectively.

This study had several limitations. This was a retrospective study based on medical records, and because of the limited number of patients, the background characteristics of patients in the IR and non-IR groups were not standardized. Future studies should aim to evaluate more cases and evaluate patients at multiple institutions. Another limitation of our study is that since most of the patients were outpatients undergoing chemotherapy, we may not have accurately assessed the infusion reactions that occurred after the patients returned home.

\section{Conclusions}

Our study indicates that the concomitant use of pertuzumab in Tra+Per combination therapy increases the risk of infusion reactions caused by trastuzumab, and the risk of infusion reactions is higher in patients with higher lymphocyte counts. Our findings can facilitate assessment of the risk of infusion reaction before administration and allow more appropriate use of the Tra+Per combination therapy according to the individual risk.

\section{Declarations}

Author contributions: All authors contributed to the conception and design of the study. Data collection and analysis were performed by Yusuke Tabuchi and Kosuke Yamamoto. The first draft of the manuscript was written by Yusuke Tabuchi, and all authors commented on the previous versions of the manuscript. All authors read and approved the final manuscript.

Funding: The authors did not receive support from any organization for the submitted work.

Data availability: N/A

Page 12/15 
Ethical approval: The Medical Ethics Review Committee of Kyoto Prefectural University of Medicine (approval no. ERB-C-2013), and the Medical Ethics Review Committee of Kyoto Pharmaceutical University (approval no. E21-013) approved this study.

Consent to participate: Since this study was a retrospective study, the informed consent of the individual participants who were included in this study was waived in accordance with the Medical Ethics Review Committee of Kyoto Prefectural University of Medicine and Kyoto Pharmaceutical University.

Consent for publication: All authors consent to the publication of this manuscript in Supportive Care in Cancer.

Conflicts of interest: Department of Endocrine and Breast Surgery, to which Koichi Sakaguchi belongs, has received a scholarship donation from Chugai Pharmaceutical Co. Keisuke Shikata has received honorarium from Chugai Pharmaceutical Co. Other authors have no relevant financial or non-financial interests to disclose.

\section{References}

1. Sandra M Swain, José Baselga, Sung-Bae Kim, Jungsil Ro, Vladimir Semiglazov, Mario Campone, Eva Ciruelos, Jean-Marc Ferrero, Andreas Schneeweiss, Sarah Heeson, Emma Clark, Graham Ross, Mark C Benyunes, Javier Cortés, CLEOPATRA Study Group (2015) Pertuzumab, trastuzumab, and docetaxel in HER2-positive metastatic breast cancer. N Eng J Med 372:724-34

2. Gunter von Minckwitz, Marion Procter, Evandro de Azambuja, Dimitrios Zardavas, Mark Benyunes, Giuseppe Viale, Thomas Suter, Amal Arahmani, Nathalie Rouchet, Emma Clark, Adam Knott, Istvan Lang, Christelle Levy, Denise A Yardley, Jose Bines, Richard D Gelber, Martine Piccart, Jose Baselga, APHINITY Steering Committee and Investigators (2017) Adjuvant Pertuzumab and Trastuzumab in Early HER2-Positive Breast Cancer. N Eng J Med 377:122-131

3. Mark Wing (2008) Monoclonal antibody first dose cytokine release syndromes-mechanisms and prediction. J Immunotoxical 5:11-15

4. Adonice Khoury, Vikas Parihar, Lillie O'steen, Kartikeya Cherabuddi (2018) Pertuzumab and trastuzumab infusion related cytokine release syndrome in a chemotherapy naive patient with metastatic breast cancer. Breast J 24:383-384

5. Schwartzberg, L. S., Stepanski, E. J., Walker, M. S., Mathias, S., Houts, A. C., \& Fortner, B. V. (2009) Implications of IV monoclonal antibody infusion reaction for the patient, caregiver, and practice: results of a multicenter study. Supportive Care in Cancer, 17: 91-98.

6. Lisa M Thompson, Karen Eckmann, Bonnie L Boster, Kenneth R Hess, Laura B Michaud, Francisco J Esteva, Gabriel N Hortobágyi, Chad M Barnett (2014) Incidence, risk factors, and management of infusion-related reactions in breast cancer patients receiving trastuzumab. Oncologist 19:228-234 
7. Christine H Chung (2008) Managing premedications and the risk for reactions to infusional monoclonal antibody therapy. Oncologist 13:725-732

8. Kanda, Y. (2013). Investigation of the freely available easy-to-use software 'EZR'for medical statistics. Bone marrow transplantation, 48:452-458.

9. European Medicines Agency: Summary of product characteristics for Perjeta (pertuzumab) https://www.ema.europa.eu/en/documents/product-information/perjeta-epar-productinformation_en.pdf (Last updated May 2021)

10. U Winkler, M Jensen, O Manzke, H Schulz, V Diehl, A Engert (1999) Cytokine-release syndrome in patients with B-cell chronic lymphocytic leukemia and high lymphocyte counts after treatment with an anti-CD20 monoclonal antibody (rituximab, IDEC-C2B8). Blood 94:2217-2224

11. Werner Scheuer, Thomas Friess, Helmut Burtscher, Birgit Bossenmaier, Josef Endl, Max Hasmann (2009) Strongly enhanced antitumor activity of trastuzumab and pertuzumab combination treatment on HER2-positive human xenograft tumor models. Cancer Res 69:9330-9336

12. Charles L Vogel, Melody A Cobleigh, Debu Tripathy, John C Gutheil, Lyndsay N Harris, Louis Fehrenbacher, Dennis J Slamon, Maureen Murphy, William F Novotny, Michael Burchmore, Steven Shak, Stanford J Stewart, Michael Press (2002) Efficacy and safety of trastuzumab as a single agent in first-line treatment of HER2-overexpressing metastatic breast cancer. J Clin Onco/ 20:719-726

\section{Figures}

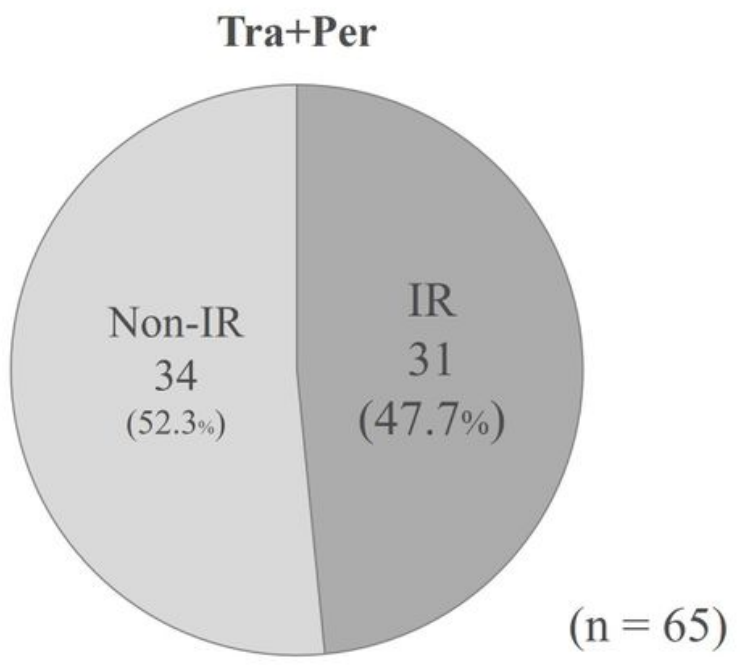

Figure 1

Fisher's exact test was used to compare the two groups $\left({ }^{\star} P<0.05\right)$. 
Tra, Trastuzumab; Per, Pertuzumab

Page 15/15 